\title{
Allostatic Mechanisms of Opioid Tolerance Beyond Desensitization and Downregulation
}

Catherine M. Cahill ${ }^{1}$, Wendy Walwyn ${ }^{3}$, Anna M.W. Taylor ${ }^{3}$, Amynah A.A. Pradhan ${ }^{2}$ and Christopher J. Evans ${ }^{3}$

${ }^{1}$ Department of Anesthesiology and Perioperative Care, University of California, Irvine 837 Health Sciences Road, Irvine, California, USA, 90095

2 Department of Psychiatry, University of Illinois at Chicago, 1601 W Taylor St., Chicago IL 60612.

${ }^{3}$ Hatos Center for Neuropharmacology, Department of Psychiatry and Biobehavioral Sciences, Semel Institute for Neuroscience and Human Behavior, University of California, Los Angeles 675 Charles E Young Drive South, Los Angeles, California, USA, 90095

*Correspondence: cevans@ucla.edu (C.J. Evans)

Key words: mu opioid receptor, arrestin, bias signaling, microglia, opponent processes, reward, dopamine

\author{
Abstract \\ Mechanisms of opioid tolerance have focused on adaptive modifications within cells containing \\ opioid receptors, defined here as cellular allostasis, emphasizing regulation of the opioid \\ receptor signalosome. Here we will review additional regulatory and opponent processes \\ involved in behavioral tolerance, and include mechanistic differences both between agonists \\ (agonist bias), and between mu and delta opioid receptors. In a process we will refer to as pass- \\ forward allostasis, cells modified directly by opioid drugs impute allostatic changes to \\ downstream circuitry. Because of the broad distribution of opioid systems, every brain cell \\ maybe touched by pass-forward allostasis in the opioid-dependent/tolerant state. We will \\ implicate neurons and microglia as interactive contributors in the cumulative allostatic \\ processes creating analgesic and hedonic tolerance to opioid drugs.
}




\section{What is Tolerance?}

Tolerance (see Glossary) is defined as a reduction in effect following prolonged drug administration resulting in a loss of drug potency indicated by a pharmacological shift to the right in the dose response curve. The development and extent of tolerance is dependent on the drug interactions with the opioid receptor(s), dose, and frequency of administration. There are many mechanisms that can contribute to opioid tolerance at a behavioral level, including upregulation of drug metabolism (metabolic tolerance), desensitization of receptor signaling, and down regulation of receptors as well as the initiation of compensatory/opponent processes. Analgesic tolerance is not always noteworthy, as many physicians argue that analgesic tolerance can simply be overcome by increasing the opioid therapeutic dose to maintain patient satisfaction or by implementing opioid rotation. However, this is not always feasible as other pharmacological effects, such as constipation, can limit patient compliance/satisfaction, because opioid-induced gut motility exhibits minimal tolerance compared to other effects, such as sedation and nausea. Moreover, management of opioid tolerant patients during acute episodes of care is also a challenge amongst health care providers, as these patients have a significantly longer length of hospital stay and a greater 30 day re-admission rate, after adjusting for risk assessment including risk of mortality (APR-DRG 3M model) and a number of comorbid conditions [1]. Furthermore, increasing the dose of opioids can precipitate or exacerbate opioid-induced hyperalgesia, which in turn contributes to behavioral analgesic tolerance and may be misdiagnosed as disease progression-induced pain. In addiction medicine, tolerance is a key component of dependence and addiction liability. The USA Food and Drug Administration (FDA) defines tolerance as $60 \mathrm{mg}$ morphine equivalent daily, and prescriptions 
of over $100 \mathrm{mg}$ morphine equivalent daily are often subject to scrutiny for opioid abuse [2]. Importantly, tolerance to opioid-induced respiratory depression may be seen as beneficial for pain patients, but can cause an increase mortality when opioid addicts take the same dose of opioid in a different environment/context [3] or relapse following a period of abstinence. For this latter population, tolerance has abated during this drug-free period and relapsing with a pre-abstinence opioid dose triggers life-threatening consequences. Another reason tolerance is central in addiction medicine is that treating pain in opioid addicts is exceedingly challenging, where methadone-maintained patients are often refractory to analgesic effects of opioid therapies due to excessive tolerance [4]. In this review, we will consider behavioral tolerance mechanisms to include drug-induced adaptations or allostatic changes at the cellular, circuitry, and system levels that require increased opioid drug to achieve the same effect. We argue that opioids generate opponent processes not only in nociceptive circuits, but also in circuits modulating mood, which contribute to the genesis of an addicted state via tolerance to the hedonic aspects of drug taking. The purpose of this review is to take a neurobiological journey, which begins with agonist-dependent regulation of the opioid receptor signalosome mediating allostatic changes within opioid-receptor containing cells, that leads to pass-forward allostasis modifying circuits and networks to create behavioral tolerance.

Opioid drugs such as morphine and fentanyl activate opioid receptors, a family of G-protein coupled receptors that primarily signal through heterotrimeric $\mathrm{G}$ protein $\mathrm{G}_{\mathrm{i} / \mathrm{o}}$ subunits. On agonist binding, opioid receptors activate a signalosome to inhibit adenylate cyclase and calcium channels, whilst activating potassium channels and several kinase cascades such as 
extracellular signal-regulated kinase (ERK1/2), c-Jun N-terminal kinases (JNK's) and AKT or

Protein Kinase B. The hallmark outcome of acute opioid agonist treatment is that neurons containing opioid receptors are less excitable, thereby influencing circuit dynamics. There are four members of the opioid receptor family in vertebrates (mu, delta, kappa and nociceptin/orphanin FQ), and activation of all four can produce analgesia, whereas substance abuse is primarily associated with activation of mu opioid receptors (MOP)[5]. Because of their differential cellular and regional distributions, agonists to the different opioid receptors elicit disparate pharmacological effects. The MOPs mediate the canonical effects of clinically used opioids, namely analgesia, euphoria, respiratory depression, and constipation. Activation of the delta opioid receptor (DOP) also produces analgesia in chronic pain states, and agonists are being developed by industry for the treatment of anxiety and migraine [6, 7]. Kappa opioid receptor (KOP) agonists elicit anxiogenic and dysphoric effects and are notable for exhibiting the opposing effects to MOP agonists on emotional regulation [8]. Industry development of KOP antagonists are being vigorously pursued for their ability to alleviate the negative affect in addiction and substance abuse disorders, whereas there is much interest in developing a peripherally acting KOP agonist for pain relief $[9,10]$. The nociceptin/orphanin FQ receptor (NOP) is the most abundant member of the opioid receptor family in brain and affects opioid analgesia, tolerance development, and reward via modulating MOP effects [11]. In addition to different receptor types, different drugs acting at the same receptor can cause different conformational changes resulting in the coupling of the receptor to different intracellular signaling cascades that produce distinct pharmacological effects. This phenomenon, referred to 
as biased agonism, is well known to occur for various opioid receptor types (for review see Pradhan et al $[12,13])$.

\section{Cellular Allostasis and the Opioid Receptor Signalosome (MOP and DOP)}

Mechanisms of opioid receptor desensitization and resensitization have been thoroughly reviewed for different agonists at the MOP [13]. The ability of different agonists to induce receptor internalization has been shown to affect divergent downstream regulatory processes contributing to the activity of the opioid receptor signalosome and cellular tolerance (see Figure 1 and legend). Many other processes contribute to cellular allostasis. MOP signaling initiates a cascade of cellular adaptations involving perturbation of kinase pathways and protein complexes [as reviewed in depth by 14]. In turn, these perturbations mediate changes in transcriptome and proteome profiles, as well as morphological changes, for instance in spine remodeling [15]. Cellular allostasis from continued opioid treatment is substantial, although not all adaptations triggered by MOP activation will contribute to tolerance. One well-studied opponent system that undoubtedly contributes to cellular tolerance is adenylate cyclase supersensitivity. Both DOP and MOP agonists inhibit adenylate cyclase thereby decreasing cellular cAMP levels. Sustained agonist stimulation is accompanied by a compensatory upregulation of both basal and stimulated cAMP levels. Mechanisms that account for this adaptation include regulation of the levels of different adenylate cyclase isoforms and phosphokinase A (PKA) [16]. In addition, a noncanonical pathway involving a switch from the classical Gi/Go coupled signaling to one involving src has been proposed. This leads to Raf1 recruitment and phosphorylation of specific cyclase isoforms to increase cAMP levels [17]. 
Many cellular adaptations occur as a result of protracted signaling via opioid receptors modifying not only the signalosome, but also the transcriptome and proteome to affect the structural integrity, connectivity, and activity of opioid receptor-containing neurons [16].

\section{The Complex Role of Arrestins in Behavioral Tolerance to Opioids}

Following the initial report that mutant mice lacking arrestin 3 (beta-arrestin 2) exhibit attenuated tolerance to morphine [18], there is now a plethora of studies demonstrating the involvement of arrestins in opioid receptor desensitization and tolerance. Both high and low internalizing MOP and DOP agonists induce analgesic tolerance [19-22] albeit via different mechanisms. Moreover, MOP receptor agonists independent of their internalization efficacy, exhibit comparable symptoms of physical withdrawal [20]. That behavioral tolerance and withdrawal is comparable between arrestin or internalization biased agonists suggests that MOP agonists regardless of mechanisms of cellular tolerance trigger overlapping allostatic circuitry regulating behavior. There are several explanations for the modulation of tolerance by arrestins. At face value, the classical mechanism would be that arrestin binding, as the name would imply, blocks G-protein signaling and promotes clatherin-mediated endocytosis. However, attenuated signaling or cellular tolerance is the sum of multiple processes including: receptor desensitization, receptor resensitization, receptor internalization (recycling or downregulation), de novo receptor synthesis and golgi stability, as well as receptor trafficking to the cell membrane (externalization). Modification by arrestins at any juncture in the receptor signalosome regulatory machinery could influence cellular and behavioral opioid tolerance. Opioid agonists can differentially recruit arrestin-dependent signaling cascades to regulate 
opioid tolerance in a ligand- and tissue- biased manner. For example, morphine analgesic tolerance is attenuated in the absence of arrestin 3, whereas that of fentanyl is not, despite both agonists being able to recruit arrestin 3 (Figure 1). Arrestin 3 in the colon appears to be necessary for tolerance to the MOP agonist DAMGO, but not fentanyl or etorphine, despite all 3 agonists effectively inducing MOP internalization [23]. It was also found that c-Jun $\mathrm{N}$-terminal kinase (JNK2) can regulate MOP tolerance and recruitment of this molecule can be arrestindependent or independent, depending on the MOP agonist used [24].

As illustrated in Table 1 and Figure 1, arrestin isoforms are a major modifier of DOP tolerance. Furthermore, arrestins are key to ligand bias of DOP agonists to trigger different intracellular signaling cascades and functional outcomes [25]. In its resting state DOP appears to form a complex with arrestin 3 and high-internalizing DOP agonists preferentially recruit arrestin 2 (Beta-arrestin 1), resulting in DOP desensitization and acute behavioral tolerance. Thus, knockout of arrestin 2 results in a dramatic increased potency and decreased acute behavioral tolerance to high-internalizing DOP agonists, such as SNC80 [19, 26] (Figure 1). In contrast, lowinternalizing DOP agonists preferentially recruit arrestin 3 and show no phenotype in the arrestin 2 knockout. In the arrestin 3 knockout, there is no phenotype for high internalizing agonists. However, for low internalizing agonists, such as ARM390 and JNJ20788560, arrestin 3 facilitates the rate of receptor resensitization. Hence, in complete contrast to the MOP, knockout of arrestin 3 resulted in increased tolerance to low-internalizing DOP agonists and reduced rates of DOP resensitization [19]. This data for MOP and DOP reveal a role of arrestins to regulate receptor resensitization, although they are traditionally considered for their role in 
mediating desensitization (Figure 1). The mechanisms of desensitization and resensitization by low-internalizing opioid agonists are currently unknown, although kinase/phosphatase activity is a likely component and PKC and JNK isoforms are implicated for MOP [24, 27]. We speculate that arrestin 3 containing complexes attenuate recruitment of relevant phosphatases for MOP resensitization, but facilitate the recruitment of relevant phosphatases for DOP resensitization.

As a part of multi-faceted protein complexes, the arrestins play important roles in opioid receptor signaling independent of ligand-induced receptor desensitization, resensitization, and internalization. For example, both arrestin 2 and 3 control the cytoskeletal protein actin by binding to cofilin and its inactivating kinase, Lim Domain Kinase, and activating phosphatases, slingshot or chronophin [28-30]. Relevant to DOP and nociceptin/orphanin FQ receptor function is that arrestin 2 acts as a chaperone controlling the rate of ligand-dependent export of these opioid receptors to the cell membrane [27]. Furthermore, a c-Src/arrestin 3 complex regulates MOP to promote ligand-independent or constitutively active MOP signaling [31]. In this complex, arrestin constrains c-Src activity by keeping this tyrosine kinase in an inactive state. Together these interactions demonstrate that the arrestins regulate opioid receptor function as part of catalytically active scaffolding complexes.

Given that arrestins are key components of opioid receptor signaling and trafficking, they are well-situated to contribute to the changes that initiate cellular allostasis, and subsequent passforward system-wide allostasis. Because of the arrestin-dependent processes involved in MOP signaling and tolerance there has been a drive to develop drugs that activate MOP without 
recruiting either arrestin 2 or 3 . Such drugs would be anticipated to have increased analgesia (possibly either promoting recycling or inducing constitutive activity) and reduced respiratory depression [32]. However, on the negative side, arrestin deletion increased constipation, a major issue with opioid drugs. Recently, clinical trials have begun on TRV130, a MOP agonist that elicits very low arrestin recruitment. There are some promising results with this drug. TRV130 has increased analgesic potency compared with morphine (1.5mg TRV130 given intravenously had an equivalent analgesic effect to $10 \mathrm{mg}$ morphine). At some time-points, the ratio of respiratory depression to analgesic efficacy is less than morphine, implying better safety (therapeutic index) profile [33] However, the pharmacokinetic differences confound therapeutic advantage over morphine, since TRV130 appears to have a shorter duration of action in both respiratory depression and analgesia. This is clearly an area of agonist bias drug development that could have positive outcome, although at this point it is unclear if MOP agonists that do not recruit arrestins at all will elicit increased gut immobility and/or altered addiction liability. The assessment of such drugs in self-administration paradigms and relapse models will provide valuable insight for whether biased ligands produce less addictive-like behaviors compared to current opioid therapeutics.

\section{Opponent Processes Contribute to a Tolerant State}

Behavioral opioid tolerance is most often studied in the context of analgesia and cellular signaling mechanisms, as reviewed above. However, opponent processes are also evident at a circuit-level in the regulation of mood and affect. In this section, we will focus on how chronic opioid exposure causes adaptations of the mesocorticolimbic circuitry due to the development 
of opponent processes in the striatum and ventral tegmental area (VTA). The striatum is an important hub of the opioid reward pathway receiving dense dopaminergic innervation from the midbrain (such as the VTA), as well as excitatory input from the basolateral amygdala, prefrontal cortex, ventral hippocampus and paraventricular thalamic nucleus that drive opioid reward seeking behaviors [34-36]. The striatum also has high intrinsic expression and projection input of endogenous opioids and expresses opioid receptors in various cell types including dopamine D1 receptor (prodynorphin)- and D2 receptor (proenkephalin)-expressing striatal medium spiny projection neurons (Figure 2). Importantly, the VTA and striatum undergoes synaptic adaptations that initiate opponent processes in response to chronic opioid use with implications for substance abuse and co-morbid affective disorders.

\section{Allostatic Processes in the Ventral Tegmental Area}

Dopamine release from VTA dopaminergic neurons reinforces natural rewarding behavior and attributes motivational salience to otherwise neutral environmental stimuli or unanticipated salient stimuli $[34,37,38]$. Dopaminergic neurons from the VTA exhibit a rich and complex organization with widespread projections to forebrain targets. [39-43]. The rewarding effects of morphine are mediated via activation of MOP, as mice lacking this receptor do not express conditioned place preference and do not self-administer opioid drugs [44]. MOP in the VTA elicits rewarding effects via disinhibition of GABAergic neurons to stimulate dopamine release [45]. Disinhibition of VTA dopaminergic neurons is primarily driven by effects of MOP on GABA input from the rostral tegmental nucleus (RMTg), rather than VTA GABAergic interneurons or GABA input from the nucleus accumbens (NAC) [46]. However, expression of MOP on 
D1/prodynorphin medium spiny projection neurons is sufficient to produce opioid reward [47]. Following chronic morphine, MOP desensitization on VTA input from the RMTg, rather than NAc or VTA GABAergic interneurons are responsible for the loss of MOP-induced disinhibition of dopamine neurons [46]. Interestingly, opioid reward in naïve animals (non-opioid dependent) does not rely on dopamine $[48,49]$, whereas dopamine signaling is required for expression of reward in opioid-dependent animals, as evidenced by systemic blockade using a non-specific dopamine antagonist [50-53]. It should be emphasized that opioid-induced reward is dependent on dopamine in opioid dependent states, despite chronic morphine causing a hypofunction of the dopaminergic mesolimbic circuitry $[54,55]$. This dopaminergic hypofunction is thought to contribute to the negative affect associated with opioid dependence possibly a result of increased KOP function.

KOPs are present on dopaminergic terminals in the NAc and inhibit dopamine release (Figure 2). They are activated by endogenous release of neuropeptides derived from prodynorphin, and produce behavioral phenotypes indicative of negative emotional states such as place aversion and depressive-like affective behaviors [56]. The prominent neuromodulatory effect of KOP on the dopaminergic system is now the subject of clinical development, with the goal of removing opioid-induced protracted abstinence syndrome characterized by negative affect that can drive relapse. There is convincing evidence that KOP in the NAc and extended amygdala drive stressinduced dysphoria and vulnerability to drug relapse. Indeed, elimination of KOP in amygdala neurons projecting to the bed nucleus of the stria terminalis [57] or within dopamine neurons [58] produced an anxiolytic phenotype. This suggests that KOP expression can contribute to 
negative affect and identifies a novel treatment strategy for mood disorders such as anxiety and depression. Additionally, KOP in the VTA also drive aversive behavior as conditional knockout of KOP from dopaminergic neurons blocked KOP agonist place aversion [59]. Further research is necessary to identify the circuitry important for the genesis of a protracted abstinence syndrome following chronic opioid treatment and whether KOP modulation can eliminate this negative affect [60].

Brain-derived neurotrophic factor (BDNF) is a critical modulator of VTA dopamine neuronal activity in opioid-dependent animals [61-63], and impacts VTA neuronal activity by shifting the $\mathrm{GABA}_{A}$ receptor reversal potential in VTA GABAergic interneurons [64]. Evidence from other systems have shown that BDNF decreases the $G_{A B A}$ receptor reversal potential via downregulation of the $\mathrm{K}+/ \mathrm{Cl}^{-}$co-transporter $\mathrm{KCC}$ [65-68], which depletes the $\mathrm{Cl}^{-}$electrochemical gradient, effectively reducing $\mathrm{GABA}_{A}$-mediated hyperpolorization (for review: [69]). Because GABAergic, but not dopaminergic, neurons express KCC2 [70], this population is particularly affected by the BDNF-driven hyperexcitability in opioid dependence. We hypothesize that this increased excitability of GABAergic neurons impairs the ability of opioid agonists to inhibit GABAergic neurons. While neurons release BDNF, we have proposed that activated microglia is the source of BDNF during abstinence following prolonged morphine administration (Box 1). Interestingly BDNF injections reverse changes in VTA dopamine soma size that are observed following chronic opioids, and suggests multi-faceted effects of BDNF on allostatic processes triggered by chronic opioids [71]. 


\section{Allostatic Processes in Striatal Cells, Signaling and Circuits}

Similar to other drugs of abuse, chronic morphine induces extensive and region-specific synaptic plasticity within the striatum. Glutamate homeostasis is affected by changes in glutamate release, re-uptake and the composition, number and role of ionotropic and metabotropic receptors (for review see [72]). At the level of the synapse, a 10 day period of withdrawal from chronic non-contingent (i.e. experimenter delivered) morphine increased synaptic strength, as assessed by the ratio of AMPA:NMDA currents, in the NAc shell $[73,74]$. Conversely, 14-days of withdrawal from contingent (i.e. subject-initiated) heroin impaired long term potentiation (LTP) and long term depression (LTD) formation in the NAc core [75]. These differences could be region-specific or a result of experimenter vs self-administered drug delivery. Opioid-induced synaptic plasticity is closely related to changes in dendritic spine density showing similar regional and drug-regimen effects. For example; spine density in the orbito-frontal cortex showed a greater increase following self-administered than experimenterdelivered morphine. In this same study, both contingent and non-contingent opioid administration decreased spine density in the NAc shell [76]. Heroin self-administration has also been shown to reduce spine density in the NAc core, but this recovered following heroin reinstatement and was accompanied by increased GluN2B-NMDARs. Interestingly blocking these receptors prevented heroin-seeking behaviors [77]. Although experimenter delivered morphine also increased GluN2B-NMDARs, the effect of this opiate differed from heroin resulting in an increased spine density in the same region [78]. Together these findings suggest that the effect of opioids on spine morphology parallel changes in synaptic plasticity and possibly GluN2B-NMDARs. These alterations in glutamatergic input will undoubtedly affect 
signaling within the striatum and striatal output. Indeed striatal neurons show increased intrinsic excitability [79] which would induce neuron-specific adaptations in regions such as the pallidum, that receive striatal input. However, it is important to note that the role of drugseeking vs drug exposure per se and region specificity must be considered when identifying opioid-induced allostatic changes in circuitry.

Studies of immediate early gene expression further demonstrate how administration of morphine not only affects MOP expressing cells, but also circuits that interact with these neurons. Thus, the effect of morphine can spread across the striatum and associated regions. A single morphine injection initially activates neurons in the dorsomedial striatum and NAc shell, but after $4 \mathrm{~h}$ activity is observed across the dorsal striatum and neocortex [80]. Co-labeling with markers of D1 and D2 medium spiny neurons (MSNs) shows that a single morphine injection increases CFOS in D1 MSNs within the NAc, whereas naloxone precipitated withdrawal activates cFOS in D2 MSNs [81]. Optogenetic experiments further define the role of these neuronal subtypes, with D1, but not D2, MSNs mediating tolerance and reward $[82,83]$. Transcriptional and proteomic profiles outline morphine-induced changes in phosphorylation cascades, energy status, and cell morphology $[84,85]$. It is unclear if the cellular adaptations observed in the striatum are restricted to opioid receptor-expressing cells or are a result of pass-forward allostasis (Box 2).

\section{Opioid-Mediated Aversion Circuitry}


The negative affect and "anti-reward" that is created by an opioid-dependent state is now recognized as a driving contributor to drug-seeking behaviors [86-88], and the neurocircuitry of the learned association between drug-induced relief of these aversive states may be particularly important for opioid substance abuse disorder. Repeated, or chronic use of opioids induces adaptive or allostatic changes that modify neuronal circuitry and create an altered normality - the "drug-dependent" state [89]. The striatum, and in particular the shell of the NAc, is often associated with reward-processing in the acute and subsequently opioid dependent, or reward-deficient, aspects of drug addiction. However, there are several projections to the striatum that have been implicated in aversive responses during withdrawal, revealing opponent processes developed during chronic drug treatments. Striatal expression of dynorphin and corticotropin-releasing factor (CRF), the proto-typical stress-associated signaling molecules, are increased by morphine withdrawal [90]; and inhibition of either system reverses chronic drug-seeking behavior [90, 91]. The modulatory role of dynorphin and KOPs in the VTANAc pathway has been discussed in the section on allosteric modulation of the VTA. The recruitment of the central and basolateral amygdala, the bed nucleus of the stria terminalis, the hippocampus and the hypothalamus have all been implicated in producing an anti-reward state, with direct or indirect consequences on the functioning of NAc circuitry. Noradrenergic inputs from the caudal medulla into the bed nucleus of the stria terminalis are also critical for opioid withdrawal-induced aversion [92].

Figure 2 outlines how chronic or prolonged exposure to opioids modifies inputs to the NAc that contribute to negative affect and the anti-reward state. Several different areas of the brain are 
involved in mediating aversion associated with opioid withdrawal. For example, withdrawalinduced aversion has been shown to involve a thalamic - NAc pathway from the paraventricular nucleus to D2-expressing MSNs of the NAc [93]. In this study, chronic morphine increased the excitatory input to NAc D2 MSNs via insertion of GluA1-containing AMPA receptors. Inhibition of this activity reversed the somatic symptoms of morphine withdrawal and naloxone precipitated withdrawal place aversion. The contribution of glutamatergic inputs to the NAc is also evidenced by the ability of micro-injections of a GluA1 antagonist in the NAc shell to inhibit naloxone conditioned place aversion [94]. Inputs from the basolateral amygdala to the NAc appear to control opioid reward by activating D1 MSNs in the opioid naïve state, followed by D2 MSNs in the opioid-dependent state [95]. These basolateral amygdala inputs to the NAc are modulated by cannabinoid 1 (CB1) receptors which can modulate the activity of either fast spiking interneurons or MSNs within the NAc shell to potentiate reward or induce aversion [96, 97]. Another glutamatergic innervation to the NAc that plays a role in opioid withdrawal and aversion are those form the infralimbic cortex. Activating the infralimbic cortex projections to the NAc shell blocks morphine reinstatement and reverses the effects of morphine abstinence [98]. There is also evidence that glutamatergic projections from the VTA to the NAc may drive aversion by activating parvalbumin GABAergic interneurons, which in turn inhibit MSNs [99]. However, the role of this projection in opioid aversion has also not been defined. The lateral habenula, an important site of aversion, reduces striatal DA levels by inhibiting VTA dopamine neurons via projections to GABAergic neurons in the RMTg [100-102]. While this lateral habenula circuitry is an important component of the negative prediction error and negative affect [43], the role of this pathway in the negative affect following chronic opioids is unknown. 
Within the striatum, optogenetic activation of dorsal or ventral KOP-expressing neurons has recently been shown to drive reward or aversion respectively suggesting that, similar to MOPs and DOPs, KOPs show specific hedonic hotspots in the NAc shell $[103,104]$.Finally, orexin neurons from the lateral region of the hypothalamus that project to the NAc, implicated in the brain stress system, block both the somatic physical symptoms and increased corticosterone levels associated with opioid withdrawal [105].

Network and functional magnetic resonance imaging (fMRI) analysis has also identified widespread changes during opioid withdrawal. Adaptations in local field potentials in regions including the basolateral amygdala, NAc and pre-frontal cortex reach a new allostatic set-point 2 days following continuous morphine treatment. This widespread allostatic state was disrupted by opioid receptor blockade and the precipitation of withdrawal [106]. Studies in humans show that withdrawal from chronic morphine alters fMRI signals in many of the limbic nuclei associated with reward, including the striatum [107]. Together this research implicates the extended amygdala and striatum as playing a central role in the allostatic changes contributing to affective opponent processes that may drive addictive behaviors.

\section{Concluding Remarks}

Tolerance to opioids per se is not a reliable predictor of abuse liability but presents a challenge for treating both acute and chronic pain, and becomes a liability for addicts that relapse. Opioid tolerance is also a predictor of significantly longer length of hospital stay and readmission rates. Tolerance often masks opioid-induced hyperalgesia and contributes to the development of 
affective disorders such as anxiety and depression, where the emergence of anxiety during withdrawal can be triggered from a single acute opioid exposure [108], but is most prominent following protracted abstinence. Opponent processes in the mesocorticolimbic circuitry, especially the striatum and the VTA, contribute to the neuro-adaptations that lead to co-morbid conditions including affective disorders. In this review, we have taken a holistic view of opioid tolerance beginning with adaptations of the opioid receptor signalosome leading on to cellular allostasis and progressing to pass-forward system-wide allostasis resulting in opponent processes that counter opioid behaviors. We highlight biased agonism as a promising avenue for developing novel therapeutics that evade selective allostasis involved in some of the negative sequelae of acute and chronic opioid use. Furthermore, microglia are identified as a mediator of the development of opponent processes (negative affect and hyperalgesia) that contribute to behavioral tolerance. Future research will determine if biased agonism or blockade of neuroinflammation will have clinical relevance in the treatment of pain or opioid addiction (see Outstanding Questions).

\section{Glossary}

- Allostasis: adaptive modifications of the nervous system following chronic opioid use that creates a new stable state dependent on presence of the drug

- Brain-derived neurotrophic factor (BDNF): factor released from neurons and/or microglia that has been shown to contribute to neuronal plasticity during development and pathology, including chronic opioid exposure 
- Delta opioid receptor (DOP): member of the opioid receptor family associated with agonist-induced antidepressant and anxiolytic effects.

- Kappa opioid receptor (KOP): member of the opioid receptor family, noted for its agonist-induced dysphoria, and opposite effects to both MOP and DOP activation with regards to affect

- Long term depression (LTD): an activity-dependent decrease in the strength (or efficacy) of a neuronal synapse that can last hours or longer

- Long term potentiation (LTP): the persistent strengthening of synapses between neurons that can last hours or longer

- Mu opioid receptor (MOP): member of the opioid receptor family eliciting the hallmark effects of opioid drugs, including euphoria, analgesia, and respiratory depression

- Mesocorticolimbic circuit: The major dopaminergic pathway originating from the midbrain and projecting to the striatum and prefrontal cortical structures. It is primarily involved in mediating motivation, and adaptations in this circuit are often observed in addicted states.

- Nucleus accumbens (NAc): Region of the striatum composed of heterogenous cell populations that is a major target of dopamine projections from the VTA

- Rostral tegmental nucleus (RMTg): region immediately rostral to the ventral tegmental area rich in GABAergic neurons that project to the VTA to control dopaminergic output, and highly implicated in opioid control of VTA dopaminergic function

- Striatum: Forebrain region involved in movement and both goal-directed and habit behaviors. Receives dense glutamatergic and dopaminergic inputs from several brain 
areas including the amygdala, frontal cortex, and VTA, and projects to the basal ganglia and midbrain nuclei.

- Tolerance: reduction in drug effect following prolonged administration resulting in a loss of drug potency indicated by a pharmacological shift to the right in the dose response curve

- Ventral tegmental area (VTA): component of the mesolimbic reward system rich in dopamine projection neurons to the striatum and frontal cortical areas

- Withdrawal: the manifestation of allostatic processes developed during drug dependence that emerge following drug cessation

Box 1: Neuroinflammation and Opioid Tolerance. Chronic exposure to opioids has been shown to activate microglia and astrocytes in the spinal cord [For review, 109]. BDNF levels are increased in the spinal cord $24 \mathrm{~h}$ after morphine abstinence, which can be blocked by in vivo treatment with microglial inhibitors [65]. Inhibition of glia, and many of the downstream signaling components, including chemokines, cytokines, fractalkine, nitrous oxide, and connexin 43 are effective at restoring morphine analgesic efficacy after chronic administration [110-114]. While initial reports have implicated activated astrocytes and microglia in the development of behavioral tolerance and opioid-induced hyperalgesia, careful analysis of spinal behavioral effects of opioids identified that microglia, contribute specifically to the development of the opioid-induced hyperalgesic component of behavioral tolerance, but not drug analgesic potency [65]. This study identified a P2X4-BDNF signaling pathway from microglia to neurons that resulted in a shift in inhibitory potential in the $\mathrm{GABA}_{A}$ expressing nociceptive specific 
lamina I spinal cord neurons. Importantly, targeted genetic deletion of BDNF from microglia was sufficient to abrogate the development of morphine-induced hyperalgesia. Thus, opponent processes can also be initiated by non-neuronal mechanisms and contribute to analgesic behavioral tolerance. Microglial activation following chronic morphine treatment has also been reported in several brain regions, including the VTA, NAc, frontal cortex, and periaqueductal grey [63, 115-117]. While initial studies implicated activated microglia as modulators of centrally mediated acute morphine actions [118], subsequent studies emphasized modulation only in opioid dependent states $[63,119]$. Blocking glial activation in opioid dependent states restores mesolimbic dopamine function, alleviates withdrawal, and prevents the relapse of drug seeking behavior after prolonged drug abstinence $[63,115,117,120,121]$. Given that microglial inhibitors also reverse the modulation of KCC2 in brain, neuroinflammation appears to be a key process in generating opioid-induced allostatic changes. In Figure i, we propose a model whereby microglia are activated during opioid withdrawal and shift the electrochemical gradient of VTA GABAergic neurons via a BDNF-KCC2 mechanism. This leads to an increased inhibitory tone on dopaminergic neurons, resulting in blunted VTA-dependent reward behaviors [63].

Box 2: Pass-Forward Allostasis. It would difficult to envisage that allostatic mechanisms accompanying opioid tolerance would be restricted to neurons containing opioid receptors, given the extensive perturbation of circuits and systems following opioid drug treatment. In the case of VTA dopaminergic cells, major changes in morphology and excitability occur after chronic morphine administration, despite these neurons not expressing MOPs [71]. Changes in 
dopaminergic neurons are likely initiated by altered GABAergic inhibitory function within the VTA or RMTg, which in turn are modulated by neuroinflammation (see Box 1). Unclear at this juncture is whether opioid drugs are directly activating microglia, or if the activation occurs as a result of MOP regulated circuitry during withdrawal. Evidence for pass-forward allostasis can also be observed in patterns of kinase activation following opioid treatment. Morphine activates ERK1/2 in many areas of the nervous system, which, as described earlier in the review, is involved in opioid tolerance in several different systems. Interestingly, in many different areas of the cortex (anterior cingulate and somatosensory), opioid-induced activation of ERK1/2 is prominent only in cells adjacent to MOP-expressing cells [122]. The inference is that modifications in neuronal populations not containing opioid receptors can have perhaps greater allostatic changes than opioid receptor containing neurons initiating opioid behaviors.

\section{Figure Legend}

Figure 1: Differences in opioid receptor regulation by low and high internalizing agonists. The cartoon depicts the DOP and MOP (red receptors have lost membrane signaling ability) after activation by a high internalizing agonist; fentanyl (for MOP) or SNC80 (for DOP), and lowinternalizing agonist; morphine (for MOP) or ARM390 (for DOP). Red arrows represent processes that downregulate opioid receptors (lysosomal degradation of agonist-internalized receptors or lysosomal targeted misfolded receptors following de-nova synthesis). Green arrows represent mechanisms of re-sensitization or enhancement of receptor activity. Several membrane permeable DOP agonists and antagonists act as chaperones for DOP resulting in increased surface receptors [123]. Externalization of vesicle-stored receptors can be enabled by 
a ROCK/LIMK mechanism, which is held in check by arrestins (Ar). High-internalizing agonists of DOP and MOP recruit G protein receptor kinases (GRKs), arrestins 2 or 3 and other receptor associated proteins or RAPs (Ras-related proteins), (left side of the figure) to promote internalization as well as arrestin-dependent and vesicle-mediated signaling [124]. Once internalized the receptors are targeted either to lysosomes or recycled back to the cell surface in a resensitized, dephoshorylated $\left(-\mathrm{PO}_{3}\right)$ state. MOPs effectively recycle back to the membrane (robust green arrow) whereas DOPs are predominantly targeted to lysosomes for degradation (robust red arrow) [125], although unlike many other receptors, this appears not to require receptor ubiquitination [126]. In contrast, activation by low internalizing agonists of MOP and DOP, only weakly recruit arrestin 3 and desensitization is reported to be dependent upon other kinases such as JNK and PKC $\left(+\mathrm{PO}_{3}\right)$. Resensitization of DOP and MOPs, presumably by dephosphorylation, following low-internalizing agonist desensitization is differentially regulated by arrestin 3. In the case of MOP, arrestin 3 (Ar3 in red) attenuates resensitization [127] whereas for DOP arrestin 3 (Ar3 in green) facilitates resensitization [19].

Figure 2. Schematic cartoon of how inputs to the nucleus accumbens are modified by chronic opioids and their contribution to negative affect. This cartoon depicts sites of neuronal allostasis in the NAc, potentially contributing to negative affect following chronic opioid treatments. Dopamine neurons from the VTA project onto two types of GABAergic MSNs defined by dopamine's ability to inhibit or excite these neurons within the NAc. Excitatory MSNs are characterized by expression of dopamine D1 receptors, GABA, dynorphin and substance $\mathrm{P}$, whereas inhibitory MSNs are characterized by expression of dopamine D2 
receptors, GABA, enkephalin and adenosine A2a receptors. The NAc also receives excitatory (glutamatergic) input from the basolateral amygdala, ventral hippocampus and prefrontal cortex that drives drug reinforcement, whereas input from the paraventricular nucleus of the thalamus can drive reward or aversion associated with withdrawal $[78,102]$. Chronic morphine is known to increase or decrease LTP and LTD of some of these glutamatergic inputs, which may depend on the method of morphine administration (contingent versus non-contingent) [75]. Other inputs originate from the dorsal raphe nucleus, hypothalamus and GABAergic projection neurons from the VTA. Kappa opioid receptors (KOP) are expressed on the axon terminals of dopamine neurons, where they can inhibit dopamine release. Chronic opioid treatment increases the expression of KOP and likely contributes to dopamine hypofunction in this circuitry. Dynorphin, the endogenous agonist at KOP is released by excitatory MSNs, however, projection neurons from the amygdala and hypothalamus are also sources of striatal dynorphin. KOPs are also present on the axon terminals of these dynorphin projection neurons. Delta opioid receptors (DOP) and mu opioid receptors (MOP) are expressed on cholinergic interneurons and are involved in modulating reward and motivation, but it is unknown to what extent they contribute to opioid tolerance and opponent processes. MOPs are also present on both excitatory, D1, and inhibitory, D2, MSNs and activation of these receptors on excitatory D1 neurons are sufficient to drive reward-like behavior [39]. In contrast, DOPs appear to be expressed in D2, but not D1, enriched MSNs [128]. Synaptic insertion of GluA2-lacking AMPA receptors in D2 MSNs is implicated in mediating the aversion as a result of activation of the paraventricular-accumbens circuitry during withdrawal [78]. Abbreviations: DA, dopamine; VTA, ventral tegmental area; DYN, dynorphin; 5-HT, serotonin; ENK, enkephalin; A2a, alpha 2a 
cholinergic receptor; PFC, prefrontal cortex; PVT, paraventricular thalamic nucleus; SP,

substance P; D2R, dopamine receptor 2; D1R, dopamine receptor 1.

Table 1. The effect of arrestin deletion on ligand-induced MOP and DOP desensitization, resensitization and tolerance.

\begin{tabular}{|c|c|c|c|c|}
\hline & \multicolumn{2}{|c|}{ MOP } & \multicolumn{2}{|c|}{ DOP } \\
\hline & Internalizing & Low-internalizing & Internalizing & Low-internalizing \\
\hline $\begin{array}{c}\text { Arrestin 2 } \\
(\beta \text {-arrestin 1) } \\
\text { deletion }\end{array}$ & $\begin{array}{l}\text { No reported } \\
\text { effects on } \\
\text { desensitization, } \\
\text { resensitization } \\
\text { or tolerance }\end{array}$ & $\begin{array}{l}\text { No observed } \\
\text { effect on } \\
\text { tolerance, or } \\
\text { reported effects } \\
\text { on } \\
\text { desensitization or } \\
\text { resensitization }\end{array}$ & $\begin{array}{l}\boldsymbol{\uparrow} \text { desensitization } \\
{[26]} \\
\boldsymbol{\uparrow} \text { externalization } \\
{[26]} \\
\boldsymbol{\uparrow} \text { efficacy and } \\
\text { potency }[19,26] \\
\Downarrow \text { acute tolerance } \\
{[19]}\end{array}$ & $\begin{array}{l}\text { No effect on } \\
\text { efficacy/potency, } \\
\text { or acute tolerance } \\
{[19]}\end{array}$ \\
\hline $\begin{array}{c}\text { Arrestin 3 } \\
\text { ( } \beta \text {-arrestin 2) } \\
\text { deletion }\end{array}$ & $\begin{array}{l}\text { No observed } \\
\text { effects on } \\
\text { tolerance or } \\
\text { desensitization } \\
\text { but } \downarrow \\
\text { resensitization } \\
{[20,31,127 \text {, }} \\
129]\end{array}$ & $\begin{array}{l}\boldsymbol{\Downarrow} \text { desensitization } \\
{[27]} \\
\uparrow \text { resensitization } \\
{[127]} \\
\downarrow \text { tolerance } \\
{[18,129]}\end{array}$ & $\begin{array}{l}\text { No effect on } \\
\text { desensitization } \\
\text { and acute } \\
\text { tolerance [19] }\end{array}$ & $\begin{array}{l}\downarrow \text { resensitization } \\
{[19]} \\
\uparrow \text { acute tolerance } \\
{[19]}\end{array}$ \\
\hline
\end{tabular}

Agonists used. DOP: Internalizing; SNC80. Low-internalizing; ARM390, JNJ20788560. MOP: Internalizing; fentanyl, DAMGO. Low-internalizing; morphine. Note that tolerance mechanisms and thus arrestin dependence may differ with measured behaviors and the table above should not be generalized to behaviors not assessed in the referenced papers.

\section{Acknowledgments}


Financial support was provided by the Shirley and Stefan Hatos Foundation (CJE, AMWT, WW), Shirley Hatos (CMC), Cousins Center for Psychoneuroimmunology (AMWT), NIH K99DA040016 (AMWT), NIH DA005010 (CJE, AMWT, WW), NIHDA031243 (AAAP), DOD MR141282 (CMC), and the UIC Department of Psychiatry (AAAP).

\section{References}

1 Gulur, P., et al. (2014) Opioid tolerance--a predictor of increased length of stay and higher readmission rates. Pain Physician 17, E503-507

2 Volkow, N.D. and McLellan, A.T. (2016) Opioid Abuse in Chronic Pain--Misconceptions and Mitigation Strategies. N Engl J Med 374, 1253-1263

3 Siegel, S., et al. (1982) Heroin "overdose" death: contribution of drug-associated environmental cues. Science 216, 436-437

4 Voon, P., et al. (2015) Pain Among High-Risk Patients on Methadone Maintenance Treatment. J Pain 16, 887-894

5 Vanderah, T.W. (2010) Delta and kappa opioid receptors as suitable drug targets for pain. Clin J Pain 26 Suppl 10, S10-15

6 Gendron, L., et al. (2016) Molecular Pharmacology of delta-Opioid Receptors.

Pharmacological reviews 68, 631-700

7 Charles, A. and Pradhan, A.A. (2016) Delta-opioid receptors as targets for migraine therapy. Curr Opin Neurol 29, 314-319

8 Bruchas, M.R., et al. (2010) The dynorphin/kappa opioid system as a modulator of stressinduced and pro-addictive behaviors. Brain research 1314, 44-55

9 Chavkin, C. and Martinez, D. (2015) Kappa Antagonist JDTic in Phase 1 Clinical Trial. Neuropsychopharmacology : official publication of the American College of Neuropsychopharmacology 40, 2057-2058

10 Chavkin, C. and Ehrich, J.M. (2014) How does stress-induced activation of the kappa opioid system increase addiction risk? Biol Psychiatry 76, 760-762

11 Toll, L., et al. (2016) Nociceptin/Orphanin FQ Receptor Structure, Signaling, Ligands, Functions, and Interactions with Opioid Systems. Pharmacological reviews 68, 419-457 12 Pradhan, A.A., et al. (2012) Ligand-directed signalling within the opioid receptor family. Br J Pharmacol 167, 960-969

13 Williams, J.T., et al. (2013) Regulation of mu-opioid receptors: desensitization, phosphorylation, internalization, and tolerance. Pharmacol Rev 65, 223-254

14 Al-Hasani, R. and Bruchas, M.R. (2011) Molecular mechanisms of opioid receptordependent signaling and behavior. Anesthesiology 115, 1363-1381

15 Sugiura, H., et al. (2009) Transducing neuronal activity into dendritic spine morphology: new roles for p38 MAP kinase and N-cadherin. Neuroscientist 15, 90-104

16 Mazei-Robison, M.S. and Nestler, E.J. (2012) Opiate-induced molecular and cellular plasticity of ventral tegmental area and locus coeruleus catecholamine neurons. Cold Spring Harb Perspect Med 2, a012070

17 Zhang, L., et al. (2013) A novel noncanonical signaling pathway for the mu-opioid receptor. Mol Pharmacol 84, 844-853

18 Bohn, L.M., et al. (2000) Mu-opioid receptor desensitization by beta-arrestin-2 determines morphine tolerance but not dependence. Nature 408, 720-723 
19 Pradhan, A.A., et al. (2016) Agonist-Specific Recruitment of Arrestin Isoforms Differentially Modify Delta Opioid Receptor Function. J Neurosci 36, 3541-3551 20 Raehal, K.M. and Bohn, L.M. (2011) The role of beta-arrestin2 in the severity of antinociceptive tolerance and physical dependence induced by different opioid pain therapeutics. Neuropharmacology 60, 58-65

21 Walker, E.A. and Young, A.M. (2001) Differential tolerance to antinociceptive effects of mu opioids during repeated treatment with etonitazene, morphine, or buprenorphine in rats. Psychopharmacology 154, 131-142

22 Pradhan, A.A., et al. (2010) Ligand-directed trafficking of the delta-opioid receptor in vivo: two paths toward analgesic tolerance. J Neurosci 30, 16459-16468

23 Maguma, H.T., et al. (2012) Differences in the characteristics of tolerance to mu-opioid receptor agonists in the colon from wild type and beta-arrestin2 knockout mice. Eur J Pharmacol 685, 133-140

24 Kuhar, J.R., et al. (2015) Mu opioid receptor stimulation activates c-Jun N-terminal kinase 2 by distinct arrestin-dependent and independent mechanisms. Cell Signal 27, 17991806

$25 \mathrm{Xu}, \mathrm{C}$., et al. (2010) Serine 363 of the \{delta\}-opioid receptor is crucial for adopting distinct pathways to activate ERK1/2 in response to stimulation with different ligands. $J$ Cell Sci 123, 4259-4270

26 Mittal, N., et al. (2013) Select G-protein-coupled receptors modulate agonist-induced signaling via a ROCK, LIMK, and beta-arrestin 1 pathway. Cell Rep 5, 1010-1021

27 Mittal, N., et al. (2012) Evidence that behavioral phenotypes of morphine in beta-arr2-/mice are due to the unmasking of JNK signaling. Neuropsychopharmacology 37, 1953-1962 28 Zoudilova, M., et al. (2007) Beta-Arrestin-Dependent Regulation of the Cofilin Pathway Downstream of Protease-Activated Receptor-2. Journal of Biological Chemistry 282, 2063420646

29 Pontrello, C.G., et al. (2012) Cofilin under control of beta-arrestin-2 in NMDA-dependent dendritic spine plasticity, long-term depression (LTD), and learning. Proc Natl Acad Sci U S A 109, E442-451

30 Zoudilova, M., et al. (2010) beta-Arrestins scaffold cofilin with chronophin to direct localized actin filament severing and membrane protrusions downstream of proteaseactivated receptor-2. J Biol Chem 285, 14318-14329

31 Walwyn, W., et al. (2007) Beta-arrestin2 and c-Src regulate the constitutive activity and recycling of mu opioid receptors in dorsal root ganglion neurons. J Neurosci 27, 5092-5104 32 Raehal, K.M., et al. (2005) Morphine side effects in beta-arrestin 2 knockout mice.J Pharmacol Exp Ther 314, 1195-1201

33 Soergel, D.G., et al. (2014) Biased agonism of the mu-opioid receptor by TRV130 increases analgesia and reduces on-target adverse effects versus morphine: A randomized, double-blind, placebo-controlled, crossover study in healthy volunteers. Pain 155, 18291835

34 Smith, K.S., et al. (2011) Disentangling pleasure from incentive salience and learning signals in brain reward circuitry. Proc Natl Acad Sci U S A 108, E255-264

35 Britt, J.P., et al. (2012) Synaptic and behavioral profile of multiple glutamatergic inputs to the nucleus accumbens. Neuron 76, 790-803

36 Stuber, G.D., et al. (2011) Excitatory transmission from the amygdala to nucleus accumbens facilitates reward seeking. Nature 475, 377-380 
37 Berridge, K.C. and Robinson, T.E. (1998) What is the role of dopamine in reward: hedonic impact, reward learning, or incentive salience? Brain research. Brain research reviews 28, 309-369

38 Pignatelli, M. and Bonci, A. (2015) Role of Dopamine Neurons in Reward and Aversion: A Synaptic Plasticity Perspective. Neuron 86, 1145-1157

39 Hitchcott, P.K., et al. (1997) Enhanced stimulus-reward learning by intra-amygdala administration of a D3 dopamine receptor agonist. Psychopharmacology (Berl) 133, 240248

40 Ikemoto, S. (2003) Involvement of the olfactory tubercle in cocaine reward: intracranial self-administration studies. J Neurosci 23, 9305-9311

41 Renard, G.M., et al. (2014) Withdrawal from chronic amphetamine reduces dopamine transmission in the rat lateral septum. J Neurosci Res 92, 937-943

42 Matsumoto, M. and Hikosaka, O. (2009) Representation of negative motivational value in the primate lateral habenula. Nat Neurosci 12, 77-84

43 Matsumoto, M. and Hikosaka, O. (2007) Lateral habenula as a source of negative reward signals in dopamine neurons. Nature 447, 1111-1115

44 Matthes, H.W., et al. (1996) Loss of morphine-induced analgesia, reward effect and withdrawal symptoms in mice lacking the mu-opioid-receptor gene. Nature 383, 819-823 45 Fields, H.L. and Margolis, E.B. (2015) Understanding opioid reward. Trends in neurosciences $38,217-225$

46 Matsui, A., et al. (2014) Separate GABA afferents to dopamine neurons mediate acute action of opioids, development of tolerance, and expression of withdrawal. Neuron 82, 1346-1356

47 Cui, Y., et al. (2014) Targeted expression of mu-opioid receptors in a subset of striatal direct-pathway neurons restores opiate reward. Nat Neurosci 17, 254-261

48 Olson, V.G., et al. (2006) Role of noradrenergic signaling by the nucleus tractus solitarius in mediating opiate reward. Science 311, 1017-1020

49 Pettit, H.O., et al. (1984) Destruction of dopamine in the nucleus accumbens selectively attenuates cocaine but not heroin self-administration in rats. Psychopharmacology (Berl) 84, 167-173

50 Bechara, A., et al. (1998) A two-separate-motivational-systems hypothesis of opioid addiction. Pharmacol Biochem Behav 59, 1-17

51 Laviolette, S.R., et al. (2004) Opiate state controls bi-directional reward signaling via GABAA receptors in the ventral tegmental area. Nature neuroscience 7, 160-169

52 Laviolette, S.R., et al. (2002) Motivational state determines the functional role of the mesolimbic dopamine system in the mediation of opiate reward processes. Behav Brain Res $129,17-29$

53 Laviolette, S.R. and van der Kooy, D. (2001) GABA(A) receptors in the ventral tegmental area control bidirectional reward signalling between dopaminergic and non-dopaminergic neural motivational systems. Eur J Neurosci 13, 1009-1015

54 Taylor, A.M., et al. (2016) Mesolimbic dopamine signaling in acute and chronic pain: implications for motivation, analgesia, and addiction. Pain 157, 1194-1198

55 Taylor, A.M., et al. (2015) Microglia disrupt mesolimbic reward circuitry in chronic pain. J Neurosci 35, 8442-8450

56 Chavkin, C. and Koob, G.F. (2016) Dynorphin, Dysphoria, and Dependence: the Stress of Addiction. Neuropsychopharmacology 41, 373-374 
57 Crowley, N.A., et al. (2016) Dynorphin Controls the Gain of an Amygdalar Anxiety Circuit. Cell Rep 14, 2774-2783

58 Van't Veer, A., et al. (2013) Ablation of kappa-opioid receptors from brain dopamine neurons has anxiolytic-like effects and enhances cocaine-induced plasticity.

Neuropsychopharmacology 38, 1585-1597

59 Ehrich, J.M., et al. (2015) Kappa Opioid Receptor-Induced Aversion Requires p38 MAPK Activation in VTA Dopamine Neurons. J Neurosci 35, 12917-12931

60 Cahill, C.M., et al. (2014) Does the kappa opioid receptor system contribute to pain aversion? Front Pharmacol 5, 253

61 Koo, J.W., et al. (2012) BDNF is a negative modulator of morphine action. Science (New York, N.Y.) 338, 124-128

62 Vargas-Perez, H., et al. (2014) BDNF signaling in the VTA links the drug-dependent state to drug withdrawal aversions. J Neurosci 34, 7899-7909

63 Taylor, A.M., et al. (2016) Neuroimmune Regulation of GABAergic Neurons Within the Ventral Tegmental Area During Withdrawal from Chronic Morphine.

Neuropsychopharmacology : official publication of the American College of

Neuropsychopharmacology 41, 949-959

64 Vargas-Perez, H., et al. (2009) Ventral tegmental area BDNF induces an opiatedependent-like reward state in naive rats. Science (New York, N.Y.) 324, 1732-1734 65 Ferrini, F., et al. (2013) Morphine hyperalgesia gated through microglia-mediated disruption of neuronal Cl(-) homeostasis. Nature neuroscience 16, 183-192

66 Gagnon, M., et al. (2013) Chloride extrusion enhancers as novel therapeutics for neurological diseases. Nat Med 19, 1524-1528

67 Ting, A.K.R., et al. (2013) Infusion of brain-derived neurotrophic factor into the ventral tegmental area switches the substrates mediating ethanol motivation. The European journal of neuroscience 37, 996-1003

68 Coull, J.A., et al. (2005) BDNF from microglia causes the shift in neuronal anion gradient underlying neuropathic pain. Nature 438, 1017-1021

69 Doyon, N., et al. (2016) Chloride Regulation: A Dynamic Equilibrium Crucial for Synaptic Inhibition. Neuron 89, 1157-1172

70 Gulacsi, A., et al. (2003) Cell type-specific differences in chloride-regulatory mechanisms and GABA(A) receptor-mediated inhibition in rat substantia nigra. J Neurosci 23,8237 8246

71 Sklair-Tavron, L., et al. (1996) Chronic morphine induces visible changes in the morphology of mesolimbic dopamine neurons. Proc Natl Acad Sci U S A 93, 11202-11207 72 Kalivas, P.W. (2009) The glutamate homeostasis hypothesis of addiction. Nat Rev Neurosci 10, 561-572

$73 \mathrm{Wu}, \mathrm{X}$, et al. (2013) Effects of morphine withdrawal on the membrane properties of medium spiny neurons in the nucleus accumbens shell. Brain Res Bull 90, 92-99

$74 \mathrm{Wu}, \mathrm{X}$, et al. (2012) Potentiation of synaptic strength and intrinsic excitability in the nucleus accumbens after 10 days of morphine withdrawal. J Neurosci Res 90, 1270-1283 75 Shen, H. and Kalivas, P.W. (2013) Reduced LTP and LTD in prefrontal cortex synapses in the nucleus accumbens after heroin self-administration. Int J Neuropsychopharmacol 16, 1165-1167 
76 Robinson, T.E., et al. (2002) Widespread but regionally specific effects of experimenterversus self-administered morphine on dendritic spines in the nucleus accumbens, hippocampus, and neocortex of adult rats. Synapse 46, 271-279

77 Shen, H., et al. (2011) Heroin relapse requires long-term potentiation-like plasticity mediated by NMDA2b-containing receptors. Proc Natl Acad Sci U S A 108, 19407-19412 78 Kobrin, K.L., et al. (2015) Acquisition of morphine conditioned place preference increases the dendritic complexity of nucleus accumbens core neurons. Addict Biol $79 \mathrm{Wu}, \mathrm{X}$, et al. (2012) Potentiation of synaptic strength and intrinsic excitability in the nucleus accumbens after 10 days of morphine withdrawal. Journal of Neuroscience Research 90, 1270-1283

80 Ziolkowska, B., et al. (2015) Temporal and anatomic patterns of immediate-early gene expression in the forebrain of C57BL/6 and DBA/2 mice after morphine administration. Neuroscience 284, 107-124

81 Enoksson, T., et al. (2012) Nucleus accumbens D2- and D1-receptor expressing medium spiny neurons are selectively activated by morphine withdrawal and acute morphine, respectively. Neuropharmacology 62, 2463-2471

82 Gaspari, S., et al. (2014) Nucleus accumbens-specific interventions in RGS9-2 activity modulate responses to morphine. Neuropsychopharmacology 39, 1968-1977 83 Koo, J.W., et al. (2014) Loss of BDNF signaling in D1R-expressing NAc neurons enhances morphine reward by reducing GABA inhibition. Neuropsychopharmacology : official publication of the American College of Neuropsychopharmacology 39, 2646-2653 84 Feng, K., et al. (2013) Comparative proteomic analysis of the nucleus accumbens during extinction and reinstatement of morphine dependence. West Indian Med J 62, 210-215 85 Tapocik, J.D., et al. (2013) Neuroplasticity, axonal guidance and micro-RNA genes are associated with morphine self-administration behavior. Addict Biol 18, 480-495 86 Koob, G.F. and Le Moal, M. (2008) Addiction and the brain antireward system. Annu Rev Psychol 59, 29-53

87 Koob, G.F., et al. (2014) Addiction as a stress surfeit disorder. Neuropharmacology $76 \mathrm{Pt}$ B, 370-382

88 Koob, G.F. (2013) Negative reinforcement in drug addiction: the darkness within. Curr Opin Neurobiol 23, 559-563

89 Evans, C.J. and Cahill, C.M. (2016) Neurobiology of opioid dependence in creating addiction vulnerability. F1000Res 5

90 Papaleo, F., et al. (2007) Disruption of the CRF/CRF1 receptor stress system exacerbates the somatic signs of opiate withdrawal. Neuron 53, 577-589

91 Park, P.E., et al. (2015) Chronic CRF1 receptor blockade reduces heroin intake escalation and dependence-induced hyperalgesia. Addict Biol 20, 275-284

92 Delfs, J.M., et al. (2000) Noradrenaline in the ventral forebrain is critical for opiate withdrawal-induced aversion. Nature 403, 430-434

$93 \mathrm{Zhu}, \mathrm{Y}$. , et al. (2016) A thalamic input to the nucleus accumbens mediates opiate dependence. Nature 530, 219-222

94 Russell, S.E., et al. (2016) Nucleus Accumbens AMPA Receptors Are Necessary for Morphine-Withdrawal-Induced Negative-Affective States in Rats. J Neurosci 36, 5748-5762 95 Lintas, A., et al. (2012) Inputs from the basolateral amygdala to the nucleus accumbens shell control opiate reward magnitude via differential dopamine D1 or D2 receptor transmission. The European journal of neuroscience 35, 279-290 
96 Baharlouei, N., et al. (2015) Blockage of acquisition and expression of morphine-induced conditioned place preference in rats due to activation of glutamate receptors type II/III in nucleus accumbens. Pharmacology, biochemistry, and behavior 135, 192-198 97 Haghparast, A., et al. (2014) Cannabinoid receptors in the basolateral amygdala are involved in the potentiation of morphine rewarding properties in the acquisition, but not expression of conditioned place preference in rats. Brain research 1565, 28-36 98 Hearing, M.C., et al. (2016) Reversal of morphine-induced cell-type-specific synaptic plasticity in the nucleus accumbens shell blocks reinstatement. Proc Natl Acad Sci U S A $113,757-762$

99 Qi, J., et al. (2016) VTA glutamatergic inputs to nucleus accumbens drive aversion by acting on GABAergic interneurons. Nature neuroscience 19, 725-733

100 Root, D.H., et al. (2014) Role of glutamatergic projections from ventral tegmental area to lateral habenula in aversive conditioning. J Neurosci 34, 13906-13910

101 Velasquez, K.M., et al. (2014) The role of the habenula in drug addiction. Front Hum Neurosci 8, 174

102 Gardon, O., et al. (2014) Expression of mu opioid receptor in dorsal diencephalic conduction system: new insights for the medial habenula. Neuroscience 277, 595-609 103 Al-Hasani, R., et al. (2015) Distinct Subpopulations of Nucleus Accumbens Dynorphin Neurons Drive Aversion and Reward. Neuron 87, 1063-1077 104 Castro, D.C. and Berridge, K.C. (2014) Opioid hedonic hotspot in nucleus accumbens shell: mu, delta, and kappa maps for enhancement of sweetness "liking" and "wanting". $J$ Neurosci 34, 4239-4250

105 Laorden, M.L., et al. (2012) Hypothalamic orexin--a neurons are involved in the response of the brain stress system to morphine withdrawal. PloS one 7, e36871 106 Dejean, C., et al. (2013) Opiate dependence induces network state shifts in the limbic system. Neurobiol Dis 59, 220-229

$107 \mathrm{Chu}, \mathrm{L} . \mathrm{F} .$, et al. (2015) Acute opioid withdrawal is associated with increased neural activity in reward-processing centers in healthy men: A functional magnetic resonance imaging study. Drug Alcohol Depend 153, 314-322

108 Radke, A.K., et al. (2011) An anatomical basis for opponent process mechanisms of opiate withdrawal. J Neurosci 31, 7533-7539

109 Watkins, L.R., et al. (2009) The "toll" of opioid-induced glial activation: improving the clinical efficacy of opioids by targeting glia. Trends in pharmacological sciences 30, 581-591 110 Horvath, R.J., et al. (2010) Inhibition of microglial P2X4 receptors attenuates morphine tolerance, Iba1, GFAP and mu opioid receptor protein expression while enhancing perivascular microglial ED2. Pain 150, 401-413 111 Johnston, I.N., et al. (2004) A role for proinflammatory cytokines and fractalkine in analgesia, tolerance, and subsequent pain facilitation induced by chronic intrathecal morphine. J Neurosci 24, 7353-7365

112 Muscoli, C., et al. (2010) Counter-regulation of opioid analgesia by glial-derived bioactive sphingolipids. J Neurosci 30, 15400-15408

113 Stefano, G.B. (1998) Autoimmunovascular regulation: morphine and anandamide and ancondamide stimulated nitric oxide release. J Neuroimmunol 83, 70-76

114 Shen, N., et al. (2014) A novel role of spinal astrocytic connexin 43: mediating morphine antinociceptive tolerance by activation of NMDA receptors and inhibition of glutamate transporter-1 in rats. CNS Neurosci Ther 20, 728-736 
115 Schwarz, J.M., et al. (2011) Early-life experience decreases drug-induced reinstatement of morphine CPP in adulthood via microglial-specific epigenetic programming of antiinflammatory IL-10 expression. J Neurosci 31, 17835-17847 116 Schwarz, J.M. and Bilbo, S.D. (2013) Adolescent morphine exposure affects long-term microglial function and later-life relapse liability in a model of addiction. J Neurosci 33, 961971

117 Hao, S., et al. (2011) The role of TNFalpha in the periaqueductal gray during naloxoneprecipitated morphine withdrawal in rats. Neuropsychopharmacology : official publication of the American College of Neuropsychopharmacology 36, 664-676

118 Hutchinson, M.R., et al. (2012) Opioid activation of toll-like receptor 4 contributes to drug reinforcement. J Neurosci 32, 11187-11200

119 Zwicker, J.D., et al. (2014) Glial TLR4 signaling does not contribute to opioid-induced depression of respiration. J Appl Physiol (1985) 117, 857-868

120 Zhang, X.Q., et al. (2012) Activation of p38 signaling in the microglia in the nucleus accumbens contributes to the acquisition and maintenance of morphine-induced conditioned place preference. Brain Behav Immun 26, 318-325

121 Theberge, F.R., et al. (2013) Effect of chronic delivery of the Toll-like receptor 4 antagonist (+)-naltrexone on incubation of heroin craving. Biol Psychiatry 73, 729-737 122 Eitan, S., et al. (2003) Brain region-specific mechanisms for acute morphine-induced mitogen-activated protein kinase modulation and distinct patterns of activation during analgesic tolerance and locomotor sensitization. J Neurosci 23, 8360-8369

123 Petaja-Repo, U.E., et al. (2002) Ligands act as pharmacological chaperones and increase the efficiency of delta opioid receptor maturation. EMBO J 21, 1628-1637 124 Irannejad, R. and von Zastrow, M. (2014) GPCR signaling along the endocytic pathway. Curr Opin Cell Biol 27, 109-116

125 Eisinger, D.A. and Schulz, R. (2005) Mechanism and consequences of delta-opioid receptor internalization. Crit Rev Neurobiol 17, 1-26

126 Henry, A.G., et al. (2011) The role of ubiquitination in lysosomal trafficking of deltaopioid receptors. Traffic 12, 170-184

127 Dang, V.C., et al. (2011) Cellular morphine tolerance produced by betaarrestin-2dependent impairment of mu-opioid receptor resensitization. J Neurosci 31, 7122-7130 128 Banghart, M.R., et al. (2015) Enkephalin Disinhibits Mu Opioid Receptor-Rich Striatal Patches via Delta Opioid Receptors. Neuron 88, 1227-1239

129 Connor, M., et al. (2015) beta-Arrestin-2 knockout prevents development of cellular mu-opioid receptor tolerance but does not affect opioid-withdrawal-related adaptations in single PAG neurons. Br J Pharmacol 172, 492-500 


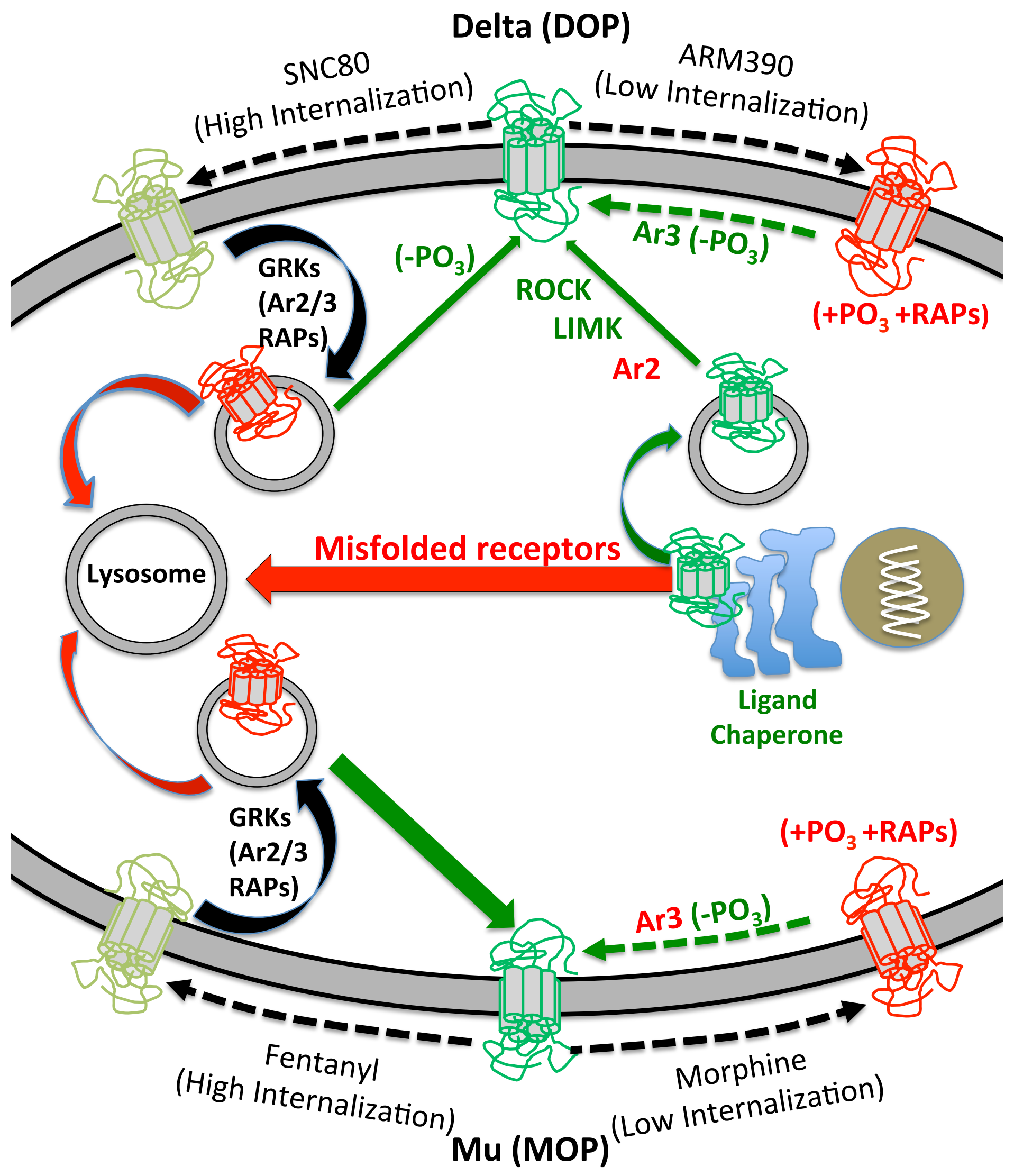




\section{$D$ DOP}

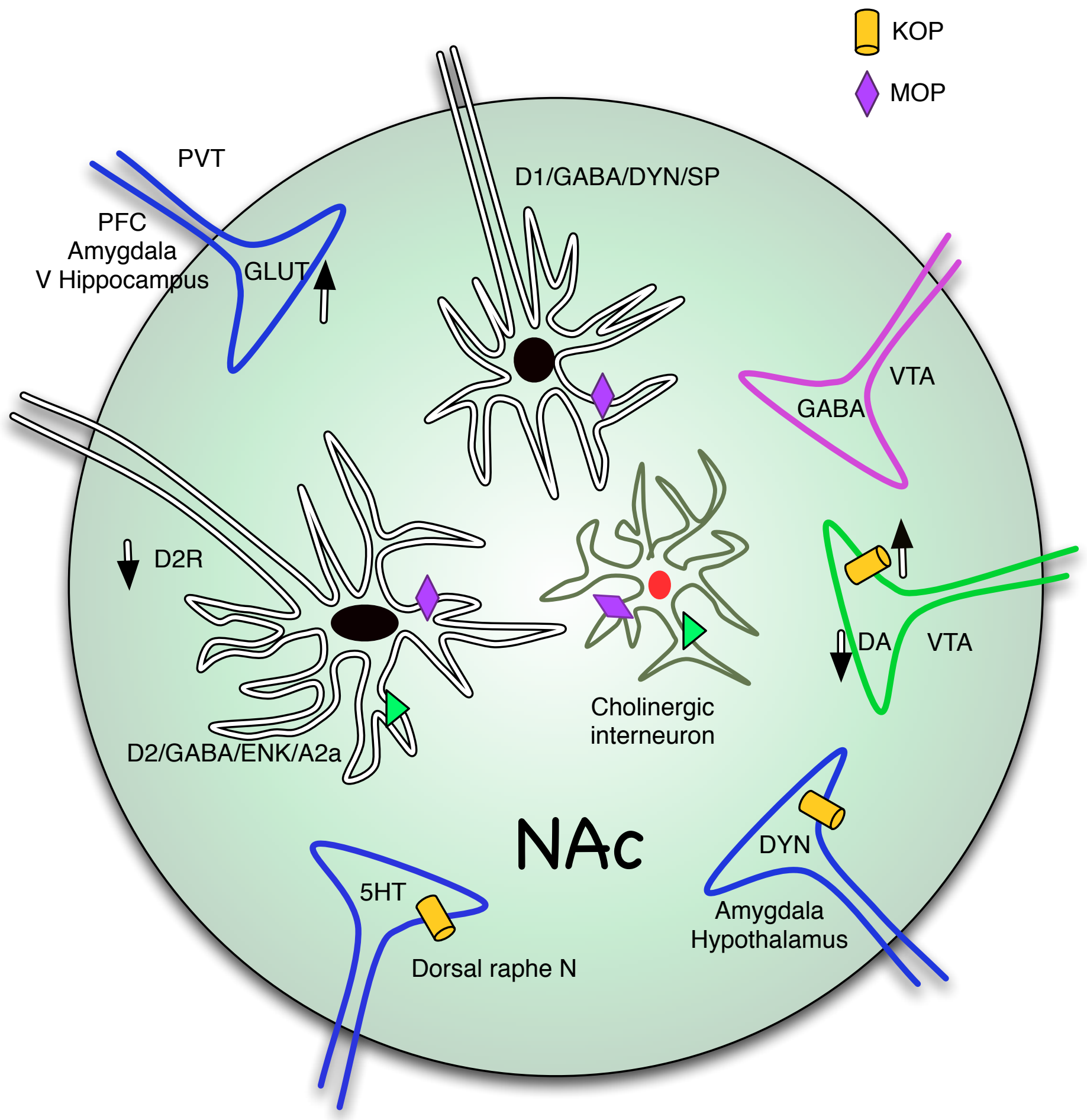


Resting Microglia

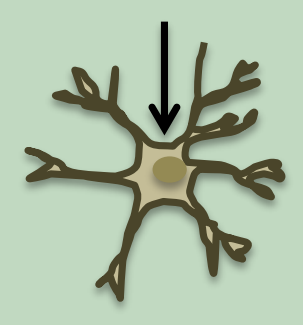

Normal
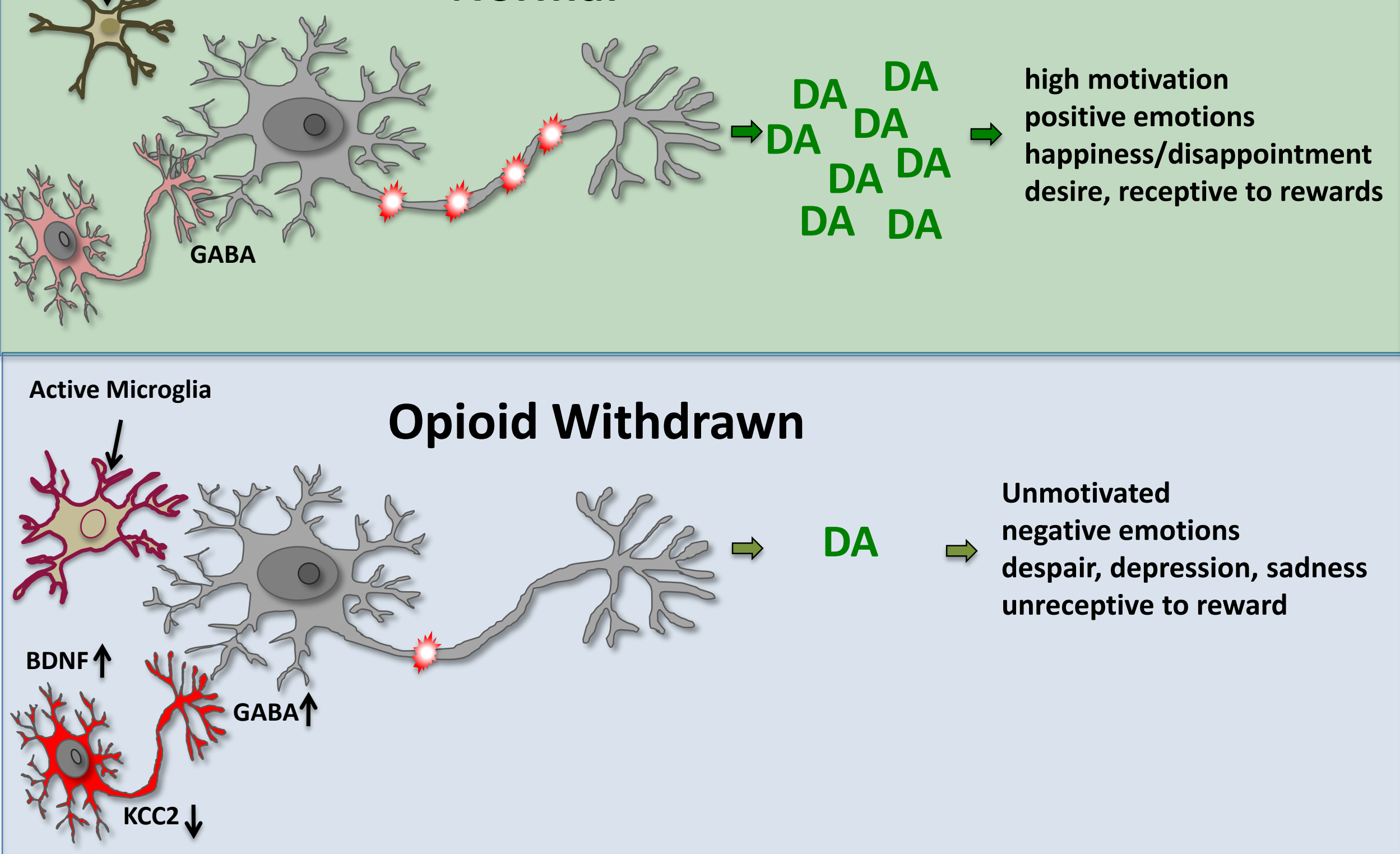

Unmotivated

DA

$\Rightarrow$ negative emotions

despair, depression, sadness

unreceptive to reward 